\title{
Development of Linearized Collision Operator for Multiple Ion Species in Gyrokinetic Flux-Tube Simulations
}

\author{
Masanori NUNAMI, Motoki NAKATA ${ }^{1, \text { a) }}$, Tomo-Hiko WATANABE ${ }^{2)}$ and Hideo SUGAMA \\ National Institute for Fusion Science, Toki 509-5292, Japan \\ 1) Japan Atomic Energy Agency, Rokkasho, Aomori 039-3212, Japan \\ ${ }^{2)}$ Department of Physics, Nagoya University, Nagoya 464-8602, Japan
}

(Received 13 February 2015 / Accepted 24 March 2015)

\begin{abstract}
Linearized model collision operators for multiple ion species are implemented in a local flux-tube gyrokinetic code. The newly implemented collision operator satisfies the conservation properties of particles, momentum, and energy, as well as the adjointness relations for collisions between different particle species, which are numerically confirmed by the test simulations. The linear zonal flow response with finite collisionality, is also compared between the new collision operator and the simplified model collision operator.
\end{abstract}

(c) 2015 The Japan Society of Plasma Science and Nuclear Fusion Research

Keywords: collision operator, gyrokinetic simulation, zonal flow

DOI: $10.1585 /$ pfr. 10.1403058

\section{Introduction}

In the magnetically confined toroidal plasma, the binary collision of charged particles is one of the most fundamental processes, and causes the classical and neoclassical transport. In the turbulent transport, the collisions are also important as the dissipative process allowing the system to reach a statistically steady state [1]. In present experiments, the magnetically confined plasma should consist of different ion species, such as deuterium, carbon, and so on. Therefore, the collisions between different ion species are necessary to be taken into account in the turbulent transport simulations of fusion plasmas. It is preferable to employ an accurate collision operator in the kinetic simulations, which is easy to treat analytically and numerically satisfying physically properties such as several conservation laws. Although several model collision operators have been proposed and numerically implemented [2-8], in this work, we implement the linearized model collision operator for multiple ion species plasmas [9] in the gyrokinetic Vlasov code, GKV [10,11]. Since the developed operator is designed for satisfying the conservation laws of particle, momentum, and energy, and physical constraints such as the Boltzmann's H-theorem and the adjointness relations, we confirm that the newly implemented collision operator works well satisfying the conservation laws, and the physical constraints within an acceptable error levels. Using the implemented collision operator, we performed a gyrokinetic simulation of the linear response of a zonal flow, where the collisional zonal flow damping process is more accurately reproduced.

The rest of this paper is organized as follows. Sec-

author's e-mail: nunami.masanori@nifs.ac.jp

a) Present address: National Institute for Fusion Science, Toki 509-5292, Japan tion 2 briefly introduces the linearized model collision operator developed in Ref. [9]. In Sec. 3, the details of the representations for the implemented collision operator into the GKV code are introduced. Section 4 is devoted to discuss the numerical tests of the operator for the conservation laws and the self-adjointness relation, and presents the gyrokinetic simulation results of zonal flow damping. Conclusions are presented in Sec. 5.

\section{The Linearized Collision Operator}

Collisional processes are necessary as the final dissipation mechanisms of the energy and entropy fluctuations in the turbulent transport induced by microinstabilities. Therefore, in the kinetic simulations, it is desirable to employ a collision operator with a set of constraints for a physically reasonable properties, an accurate collision model which preserves conservations of particles, momentum, and energy should be included. In our previous work [9], the linearized model collision operator for multiple ion species plasmas were constructed, which satisfies not only the conservation laws for particle, momentum, and energy, but also the adjointness relations and the Boltzmann's H-theorem.

\subsection{The gyrokinetic collision operator}

A well-established collision operator between plasma particle species $a$ and $b$ is given by the Landau collision operator [12] $C_{a b}\left(f_{a}, f_{b}\right)$. where the operator is bilinear with respect to the distribution functions, $f_{a}$ and $f_{b}$. If the distribution functions are given by $f_{a}=f_{a 0}+\delta f_{a}$, where $f_{a 0}$ is the equilibrium part and $\delta f_{a}$ is the small perturbation part, one may use the linearized collision operator $C_{a b}^{\mathrm{L}}$ which is defined by $C_{a b}^{\mathrm{L}}\left(\delta f_{a}, \delta f_{b}\right)=C_{a b}^{\mathrm{T}}\left(\delta f_{a}\right)+C_{a b}^{\mathrm{F}}\left(\delta f_{b}\right)$. Here, the test- 
particle part and the field-particle part of the collision operator are defined by $C_{a b}^{\mathrm{T}}\left(\delta f_{a}\right) \equiv C_{a b}\left(\delta f_{a}, f_{b 0}\right)$ and $C_{a b}^{\mathrm{F}}\left(\delta f_{b}\right) \equiv$ $C_{a b}\left(f_{a 0}, \delta f_{b}\right)$, respectively. The equilibrium part of the distribution function is assumed to be Maxwellian, $f_{a 0}=$ $F_{a \mathrm{M}} \equiv n_{a}\left(m_{a} / 2 \pi T_{a}\right)^{3 / 2} \exp \left(-m_{a} v^{2} / 2 T_{a}\right)$, where $n_{a}, m_{a}$, and $T_{a}$ are the density, the mass, and the temperature of species $a$. The perturbed particle distribution function is represented by $\delta f_{a}=\sum_{k_{\perp}} \delta f_{a k_{\perp}} \exp \left(i S_{k_{\perp}}\right)$ with the eikonal $S_{k_{\perp}}$ which describes the rapid perpendicular variation. The distribution function for the wavenumber $\boldsymbol{k}_{\perp}$ is divided into the adiabatic and non-adiabatic parts,

$$
\delta f_{a k_{\perp}}=-\frac{e_{a} \delta \phi_{k_{\perp}}}{T_{a}} F_{a \mathrm{M}}+h_{a k_{\perp}} \exp \left(-i \boldsymbol{k}_{\perp} \cdot \boldsymbol{\rho}_{a}\right),
$$

where $h_{a k_{\perp}}$ is the non-adiabatic part of the distribution function, which is independent of the gyro-phase $\varphi$, the gyro-radius vector $\rho_{a} \equiv \boldsymbol{b} \times \boldsymbol{v} / \Omega_{a}$ with $\Omega_{a} \equiv e_{a} B / m_{a} c$, and $\delta \phi_{k_{\perp}}$ is the electrostatic potential. Here, $\boldsymbol{b}, c$ and $e_{a}$ are the unit vector parallel to the magnetic field, the speed of light, and the charge of ion species $a$, respectively. The gyrokinetic form of the collision operator $C_{a b}^{(\mathrm{GK})}$ is defined by taking the gyro-phase average of the linearized collision operator as follows:

$$
\begin{aligned}
C_{a b}^{(\mathrm{GK})}\left(h_{a k_{\perp}}, h_{b k_{\perp}}\right) & \equiv \oint \frac{d \varphi}{2 \pi} \exp \left(i \boldsymbol{k}_{\perp} \cdot \boldsymbol{\rho}_{a}\right) C_{a b}^{\mathrm{L}}\left(\delta f_{a k_{\perp}}, \delta f_{b k_{\perp}}\right) \\
& \equiv C_{a b}^{\mathrm{T}(\mathrm{GK})}+C_{a b}^{\mathrm{F}(\mathrm{GK})}
\end{aligned}
$$

where the test-particle and the field-particle parts of the collision operator in gyrokinetic form are defined by

$$
\begin{aligned}
C_{a b}^{\mathrm{T}(\mathrm{GK})} & \equiv \oint \frac{d \varphi}{2 \pi} \exp \left(i \boldsymbol{k}_{\perp} \cdot \boldsymbol{\rho}_{a}\right) C_{a b}^{\mathrm{T}}\left(\exp \left(-i \boldsymbol{k}_{\perp} \cdot \boldsymbol{\rho}_{a}\right) h_{a k_{\perp}}\right), \\
C_{a b}^{\mathrm{F}(\mathrm{GK})} & \equiv \oint \frac{d \varphi}{2 \pi} \exp \left(i \boldsymbol{k}_{\perp} \cdot \boldsymbol{\rho}_{a}\right) C_{a b}^{\mathrm{F}}\left(\exp \left(-i \boldsymbol{k}_{\perp} \cdot \boldsymbol{\rho}_{b}\right) h_{b k_{\perp}}\right),
\end{aligned}
$$

respectively.

Now, we define $C_{a b}^{\mathrm{T} 0}$ as follows:

$C_{a b}^{\mathrm{T} 0}(g) \equiv v_{\mathrm{D}}^{a b} \mathcal{L}(g)+\frac{1}{2 v^{2}} \frac{\partial}{\partial v}\left[v_{\|}^{a b}(v) v^{4} F_{a \mathrm{M}} \frac{\partial}{\partial v}\left(\frac{g}{F_{a \mathrm{M}}}\right)\right]$,

where $\mathcal{L}$ is the pitch-angle-scattering operator, $\mathcal{L} \equiv$ $(1 / 2)(\partial / \partial \boldsymbol{v}) \cdot\left(v^{2} \boldsymbol{I}-\boldsymbol{v} \boldsymbol{v}\right) \cdot(\partial / \partial \boldsymbol{v})$. The collision frequencies for pitch-angle scattering and energy diffusion are given by $v_{\mathrm{D}}^{a b}(v) \equiv(3 \sqrt{\pi} / 4) \tau_{a b}^{-1}\left[H\left(x_{b}\right)-G\left(x_{b}\right)\right] / x_{a}^{3}$ and $v_{\|}^{a b}(v) \equiv(3 \sqrt{\pi} / 2) \tau_{a b}^{-1} G\left(x_{b}\right) / x_{a}^{3}$, respectively. Here, $H(x) \equiv$ $2 \pi^{-1 / 2} \int_{0}^{x} d t \exp \left(-t^{2}\right), G(x) \equiv\left[H(x)-x H^{\prime}(x)\right] /\left(2 x^{2}\right)$, $H^{\prime}(x)=2 \pi^{-1 / 2} \exp \left(-x^{2}\right), \quad x_{a} \equiv v /\left(2 T_{a} / m_{a}\right)^{1 / 2}$, and $(3 \sqrt{\pi} / 4) \tau_{a b}^{-1} \equiv 4 \pi n_{b} e_{a}^{2} e_{b}^{2} \ln \Lambda_{a b} /\left(m_{a}^{1 / 2} T_{a}^{3 / 2}\right)$, where $\ln \Lambda_{a b}$ is the Coulomb logarithm. Using the operator $C_{a b}^{\mathrm{T} 0}$, the testparticle part of the gyrokinetic collision operator can be written by

$$
\begin{aligned}
& C_{a b}^{\mathrm{T}(\mathrm{GK})}=\oint \frac{d \varphi}{2 \pi} \exp \left(i \boldsymbol{k}_{\perp} \cdot \boldsymbol{\rho}_{a}\right) C_{a b}^{\mathrm{T} 0}\left(\exp \left(-i \boldsymbol{k}_{\perp} \cdot \boldsymbol{\rho}_{a}\right) h_{a k_{\perp}}\right) \\
& +\left(\theta_{a b}-1\right) \oint \frac{d \varphi}{2 \pi} \exp \left(i \boldsymbol{k}_{\perp} \cdot \boldsymbol{\rho}_{a}\right) \boldsymbol{P}_{a} C_{a b}^{\mathrm{T} 0}\left(\exp \left(-i \boldsymbol{k}_{\perp} \cdot \boldsymbol{\rho}_{a}\right) h_{a k_{\perp}}\right) \\
& +\left(\theta_{a b}-1\right) \oint \frac{d \varphi}{2 \pi} \exp \left(i \boldsymbol{k}_{\perp} \cdot \boldsymbol{\rho}_{a}\right) C_{a b}^{\mathrm{T} 0} \mathcal{P}_{a}\left(\exp \left(-i \boldsymbol{k}_{\perp} \cdot \boldsymbol{\rho}_{a}\right) h_{a k_{\perp}}\right)
\end{aligned}
$$

$+\left(\theta_{a b}-1\right)^{2} \oint \frac{d \varphi}{2 \pi} \exp \left(i \boldsymbol{k}_{\perp} \cdot \boldsymbol{\rho}_{a}\right) \mathcal{P}_{a} C_{a b}^{\mathrm{T} 0} \mathcal{P}_{a}\left(\exp \left(-i \boldsymbol{k}_{\perp} \cdot \boldsymbol{\rho}_{a}\right) h_{a k_{\perp}}\right)$

where $\theta_{a b} \equiv\left[T_{a}\left(m_{a}+m_{b}\right) /\left(T_{a} m_{b}+T_{b} m_{a}\right)\right]^{1 / 2}$, and $\mathcal{P}_{a} g \equiv$ $F_{a \mathrm{M}}\left[\left(m_{a} / T_{a}\right) \boldsymbol{u}_{a}(g) \cdot \boldsymbol{v}+\left(\delta T_{a}(g) / T_{a}\right)\left(x_{a}^{2}-3 / 2\right)\right]$ with $\boldsymbol{u}_{a}(g) \equiv$ $n_{a}^{-1} \int d^{3} v g v$, and $\delta T_{a}(g) / T_{a} \equiv n_{a}^{-1} \int d^{3} v g\left(m_{a} v^{2} / 3 T_{a}-1\right)$. For each term in the right hand side of Eq. (6), we have more concrete expressions using the Bessel functions $J_{0}^{a}=$ $J_{0}\left(k_{\perp} v_{\perp} / \Omega_{a}\right)$ and $J_{1}^{a}=J_{1}\left(k_{\perp} v_{\perp} / \Omega_{a}\right)$ as following

$$
\begin{aligned}
& \oint \frac{d \varphi}{2 \pi} \exp \left(i \boldsymbol{k}_{\perp} \cdot \boldsymbol{\rho}_{a}\right) C_{a b}^{\mathrm{T} 0}\left(\exp \left(-i \boldsymbol{k}_{\perp} \cdot \boldsymbol{\rho}_{a}\right) h_{a k_{\perp}}\right) \\
& =v_{\mathrm{D}}^{a b}(v) \mathcal{L} h_{a k_{\perp}}+\frac{1}{2 v^{2}} \frac{\partial}{\partial v}\left[v_{\|}^{a b}(v) v^{4} F_{a \mathrm{M}} \frac{\partial}{\partial v}\left(\frac{h_{a k_{\perp}}}{F_{a \mathrm{M}}}\right)\right] \\
& -h_{a k_{\perp}} \frac{k_{\perp}^{2}}{4 \Omega_{a}^{2}}\left[v_{\mathrm{D}}^{a b}(v)\left(2 v_{\|}^{2}+v_{\perp}^{2}\right)+v_{\|}^{a b}(v) v_{\perp}^{2}\right], \\
& \left(\theta_{a b}-1\right) \oint \frac{d \varphi}{2 \pi} \exp \left(i \boldsymbol{k}_{\perp} \cdot \boldsymbol{\rho}_{a}\right) \mathcal{P}_{a} C_{a b}^{\mathrm{T} 0}\left(\exp \left(-i \boldsymbol{k}_{\perp} \cdot \boldsymbol{\rho}_{a}\right) h_{a k_{\perp}}\right) \\
& =\left(\theta_{a b}-1\right) \frac{F_{a \mathrm{M}}}{n_{a}}\left[J_{0}^{a} v_{\|} \int d^{3} v J_{0}^{a} \frac{h_{a k_{\perp}}}{F_{a \mathrm{M}}} C_{a b}^{\mathrm{T} 0}\left(F_{a \mathrm{M}} m_{a} v_{\|} / T_{a}\right)\right. \\
& +J_{1}^{a} v_{\perp} \int d^{3} v J_{1}^{a} \frac{h_{a k_{\perp}}}{F_{a \mathrm{M}}} \frac{v_{\perp}}{v_{\|}} C_{a b}^{\mathrm{T} 0}\left(F_{a \mathrm{M}} m_{a} v_{\|} / T_{a}\right) \\
& \left.+J_{0}^{a}\left(x_{a}^{2}-\frac{3}{2}\right) \int d^{3} v J_{0}^{a} \frac{h_{a k_{\perp}}}{F_{a \mathrm{M}}} \frac{2}{3} C_{a b}^{\mathrm{T} 0}\left(F_{a \mathrm{M}} x_{a}^{2}\right)\right], \\
& \left(\theta_{a b}-1\right) \oint \frac{d \varphi}{2 \pi} \exp \left(i \boldsymbol{k}_{\perp} \cdot \boldsymbol{\rho}_{a}\right) C_{a b}^{\mathrm{T} 0} \mathcal{P}_{a}\left(\exp \left(-i \boldsymbol{k}_{\perp} \cdot \boldsymbol{\rho}_{a}\right) h_{a k_{\perp}}\right) \\
& =\left(\theta_{a b}-1\right)\left[J_{0}^{a} C_{a b}^{\mathrm{T} 0}\left(F_{a \mathrm{M}} m_{a} v_{\|} / T_{a}\right) n_{a}^{-1} \int d^{3} v J_{0}^{a} h_{a k_{\perp}} v_{\|}\right. \\
& +J_{1}^{a} \frac{v_{\perp}}{v_{\|}} C_{a b}^{\mathrm{T} 0}\left(F_{a \mathrm{M}} m_{a} v_{\|} / T_{a}\right) n_{a}^{-1} \int d^{3} v J_{1}^{a} h_{a k_{\perp}} v_{\perp} \\
& \left.+J_{0}^{a} C_{a b}^{\mathrm{T} 0}\left(F_{a \mathrm{M}} x_{a}^{2}\right) n_{a}^{-1} \int d^{3} v J_{0}^{a} h_{a k_{\perp}} \frac{2}{3}\left(x_{a}^{2}-\frac{3}{2}\right)\right], \\
& \left(\theta_{a b}-1\right)^{2} \oint \frac{d \varphi}{2 \pi} \exp \left(i \boldsymbol{k}_{\perp} \cdot \boldsymbol{\rho}_{a}\right) \mathcal{P}_{a} C_{a b}^{\mathrm{T} 0} \mathcal{P}_{a}\left(\exp \left(-i \boldsymbol{k}_{\perp} \cdot \boldsymbol{\rho}_{a}\right) h_{a k_{\perp}}\right) \\
& =-\left(\theta_{a b}-1\right)^{2} \frac{F_{a \mathrm{M}}}{n_{a} \tau_{a b}} \frac{\alpha_{a b}}{\left(1+\alpha_{a b}^{2}\right)^{1 / 2}} \\
& \times\left[\frac{m_{a}}{T_{a}}\left(J_{0}^{a} v_{\|} \int d^{3} v J_{0}^{a} h_{a k_{\perp}} v_{\|}+J_{1}^{a} v_{\perp} \int d^{3} v h_{a k_{\perp}} v_{\perp}\right)\right. \\
& \left.+\frac{2 J_{0}^{a}}{1+\alpha_{a b}^{2}}\left(x_{a}^{2}-\frac{3}{2}\right) \int d^{3} v J_{0}^{a} h_{a k_{\perp}} \frac{2}{3}\left(x_{a}^{2}-\frac{3}{2}\right)\right] \text {, }
\end{aligned}
$$

where $\alpha_{a b} \equiv \sqrt{T_{a} m_{b} / T_{b} m_{a}}=x_{b} / x_{a}$.

On the other hand, the field-particle collision term is given by

$$
\begin{aligned}
& C_{a b}^{\mathrm{F}(\mathrm{GK})}=-\frac{T_{b}}{\gamma_{a b}} C_{a b}^{\mathrm{T}}\left(F_{a \mathrm{M}} m_{a} v_{\|} / T_{a}\right) \\
& \times\left[J_{0}^{a} \int d^{3} v J_{0}^{b} \frac{h_{b k_{\perp}}}{F_{b \mathrm{M}}} C_{b a}^{\mathrm{T}}\left(F_{b \mathrm{M}} m_{b} v_{\|} / T_{b}\right)\right. \\
& \left.\quad+J_{1}^{a} \frac{v_{\perp}}{v_{\|}} \int d^{3} v J_{1}^{b} \frac{h_{b k_{\perp}}}{F_{b \mathrm{M}}} \frac{v_{\perp}}{v_{\|}} C_{b a}^{\mathrm{T}}\left(F_{b \mathrm{M}} m_{b} v_{\|} / T_{b}\right)\right] \\
& -\frac{T_{b}}{\eta_{a b}} J_{0}^{a} C_{a b}^{\mathrm{T}}\left(F_{a \mathrm{M}} x_{a}^{2}\right) \int d^{3} v J_{0}^{b} \frac{h_{b k_{\perp}}}{F_{b \mathrm{M}}} C_{b a}^{\mathrm{T}}\left(F_{b \mathrm{M}} x_{b}^{2}\right),
\end{aligned}
$$


where $\eta_{a b}$ and $\gamma_{a b}$ are defined by

$$
\begin{aligned}
\eta_{a b} & \equiv-\frac{n_{a} T_{a}}{\tau_{a b}} \frac{3 \alpha_{a b}}{\left(1+\alpha_{a b}^{2}\right)^{5 / 2}}\left(\frac{T_{a}}{T_{b}}+\alpha_{a b}^{2}\right), \\
\gamma_{a b} & \equiv-\frac{n_{a} m_{a}}{\tau_{a b}} \frac{\alpha_{a b}}{\left(1+\alpha_{a b}^{2}\right)^{3 / 2}}\left(\frac{T_{a}}{T_{b}}+\alpha_{a b}^{2}\right) .
\end{aligned}
$$

\subsection{Properties}

In this subsection, we explicitly remark the several properties that the linearized Landau collision operator should satisfy for collisions between species $a$ and $b$. The conservation of particles should be satisfied for the testand field-particle parts, which are represented by

$$
\int d^{3} v C_{a b}^{\mathrm{T}}\left(\delta f_{a}\right)=\int d^{3} v C_{a b}^{\mathrm{F}}\left(\delta f_{b}\right)=0,
$$

while the momentum conservation,

$$
\int d^{3} v m_{a} v C_{a b}^{\mathrm{T}}\left(\delta f_{a}\right)+\int d^{3} v m_{b} v C_{b a}^{\mathrm{F}}\left(\delta f_{a}\right)=0,
$$

and the energy conservation,

$$
\int d^{3} v \frac{1}{2} v^{2} m_{a} C_{a b}^{\mathrm{T}}\left(\delta f_{a}\right)+\int d^{3} v \frac{1}{2} v^{2} m_{b} C_{b a}^{\mathrm{F}}\left(\delta f_{a}\right)=0,
$$

should be satisfied when each conservation is simultaneously included. The adjointness relations for the testparticle part is represented by

$$
\int d^{3} v \frac{\delta f_{a}}{F_{a \mathrm{M}}} C_{a b}^{\mathrm{T}}\left(\delta g_{a}\right)=\int d^{3} v \frac{\delta g_{a}}{F_{a \mathrm{M}}} C_{a b}^{\mathrm{T}}\left(\delta f_{a}\right),
$$

and for the field-particle operator,

$$
T_{a} \int d^{3} v \frac{\delta f_{a}}{F_{a \mathrm{M}}} C_{a b}^{\mathrm{F}}\left(\delta f_{b}\right)=T_{b} \int d^{3} v \frac{\delta f_{b}}{F_{b \mathrm{M}}} C_{b a}^{\mathrm{F}}\left(\delta f_{a}\right) .
$$

The H-theorem can be written by

$$
\begin{aligned}
& T_{a} \int d^{3} v \frac{\delta f_{a}}{F_{a \mathrm{M}}}\left[C_{a b}^{\mathrm{T}}\left(\delta f_{a}\right)+C_{a b}^{\mathrm{F}}\left(\delta f_{b}\right)\right] \\
& \quad+T_{b} \int d^{3} v \frac{\delta f_{b}}{F_{b \mathrm{M}}}\left[C_{b a}^{\mathrm{T}}\left(\delta f_{b}\right)+C_{b a}^{\mathrm{F}}\left(\delta f_{a}\right)\right] \leq 0 .
\end{aligned}
$$

Equation (19) expresses the dissipative nature of collisions which cause the distribution function to asymptotically approach the local equilibrium state. The adjointness relations and the H-theorem described by Eqs. (17)-(19) are rigorously satisfied by the linearized Landau collision operator only for the case of $T_{a}=T_{b}$ [9]. If $T_{a} \neq T_{b}$ and $m_{a} \ll m_{b}$, Eqs. (17) and (18) are valid up to the lowest order of $\left(m_{a} / m_{b}\right)^{1 / 2}$. In the case of $m_{a} \gg m_{b}$, on the other hand, the test-particle part $C_{a b}^{\mathrm{T}}$ contains the term proportional to $\left(1-T_{b} / T_{a}\right)$ that causes an error to the relation (17) and the H-theorem (19). The relative magnitude of the error in the sum of $C_{a a}^{\mathrm{T}}\left(\delta f_{a}\right)+C_{a b}^{\mathrm{T}}\left(\delta f_{a}\right)$ are of the order of $\left(n_{b} / n_{a}\right)\left(e_{b} / e_{a}\right)^{2}\left(m_{b} / m_{a}\right)^{1 / 2}\left(1-T_{b} / T_{a}\right)$. Therefore, when $\left(m_{b} / m_{a}\right)^{1 / 2}\left(1-T_{b} / T_{a}\right)$ is small enough, the term contained in $C_{a b}^{\mathrm{T}}\left(\delta f_{a}\right)$, which breaks the relations in Eqs. (17) and (18), can be neglected without influencing the solution $\delta f_{a}$.

\section{The Collision Operator in GKV 3.1 GKV code}

In our previous paper [13], we performed gyrokinetic Vlasov simulations for ion temperature gradient (ITG) turbulent transport in the Large Helical Device [14] (LHD) plasma with the high ion temperature [15] by using the gyrokinetic local flux-tube turbulence code [11] based on GKV code [10]. In the GKV, which is extended recently to multiple species plasma turbulence simulations, the wavenumber-space representation of electrostatic gyrokinetic equation for the perturbed gyrocenter distribution function $\delta f_{a k_{\perp}}$,

$$
\begin{aligned}
& \left(\frac{\partial}{\partial t}+v_{\|} \boldsymbol{b} \cdot \nabla-\frac{\mu}{m_{a}} \boldsymbol{b} \cdot \nabla B \frac{\partial}{\partial v_{\|}}+i \omega_{a \mathrm{D}}\right) \delta f_{a k_{\perp}} \\
& -\frac{c}{B} \sum_{\Delta} \boldsymbol{b} \cdot\left(\boldsymbol{k}_{\perp}^{\prime} \times \boldsymbol{k}_{\perp}^{\prime \prime}\right) J_{0}\left(k_{\perp}^{\prime} v_{\perp} / \Omega_{a}\right) \delta \phi_{k_{\perp}^{\prime}} \delta f_{a k_{\perp}^{\prime \prime}} \\
& =\frac{e_{a}}{T_{a}} F_{a \mathrm{M}}\left(-v_{\|} \boldsymbol{b} \cdot \nabla-i \omega_{a \mathrm{D}}+i \omega_{a *}\right) J_{0}\left(k_{\perp} v_{\perp} / \Omega_{a}\right) \delta \phi_{k_{\perp}}+C_{a}^{(\mathrm{GK})},
\end{aligned}
$$

is solved. Here, $\omega_{a \mathrm{D}}=\boldsymbol{k}_{\perp} \cdot \boldsymbol{v}_{a \mathrm{D}}$ and $\omega_{a *}=\boldsymbol{k}_{\perp}$. $\boldsymbol{v}_{a *}$ are the magnetic and diamagnetic drift frequencies with $\boldsymbol{v}_{a \mathrm{D}}=\left(c / e_{a} B\right) \boldsymbol{b} \times\left(\mu \nabla B+m_{a} v_{\|}^{2} \boldsymbol{b} \cdot \nabla \boldsymbol{b}\right)$ and $\boldsymbol{v}_{a *}=$ $\left(c T_{a} / e_{a} B\right) \boldsymbol{b} \times\left[\nabla \ln n_{a}+\left(m_{a} v^{2} / 2 T_{a}-3 / 2\right) \nabla \ln T_{a}\right]$, respectively. In Eq. (20), the symbol $\sum_{\Delta}$ means double summations respect to $\boldsymbol{k}_{\perp}^{\prime}$ and $\boldsymbol{k}_{\perp}^{\prime \prime}$, which satisfy $\boldsymbol{k}_{\perp}=\boldsymbol{k}_{\perp}^{\prime}+\boldsymbol{k}_{\perp}^{\prime \prime}$. Although GKV code has been extended to be applicable to the electro-magnetic turbulence simulation [16], for simplicity in this paper, we consider the low- $\beta$ electrostatic limit with assuming the adiabatic electron response. Thus, the electrostatic potential $\delta \phi_{k_{\perp}}$ is determined by Poisson's equation,

$$
\begin{gathered}
\sum_{a \neq e} \int d^{3} v Z_{a} J_{0}^{a} \delta f_{a k_{\perp}}-\sum_{a \neq e} \frac{Z_{a}^{2} e}{T_{a}} n_{a}\left(1-\Gamma_{0}^{a}\right) \delta \phi_{k_{\perp}} \\
=\frac{n_{0} e}{T_{e}} \delta \phi_{k_{\perp}}\left(k_{\perp} \lambda_{\mathrm{D}}\right)^{2}+\frac{n_{e} e}{T_{e}}\left(\delta \phi_{k_{\perp}}-\left\langle\delta \phi_{k_{\perp}}\right\rangle_{\mathrm{ZF}}\right)
\end{gathered}
$$

where $Z_{a}$ represents the charge number of the species $a$, $\left\langle\delta \phi_{k_{\perp}}\right\rangle_{\mathrm{ZF}}$ is the zonal flow component of the flux surface average of $\delta \phi_{k_{\perp}}$, and $\lambda_{\mathrm{D}}$ is the Debye length. Also, $\Gamma_{0}^{a}=$ $\exp \left(-b_{a}\right) I_{0}\left(b_{a}\right)$ with $b_{a}=\left(k_{\perp}^{2} T_{a} /\left(m_{a} \Omega_{a}\right)\right)$ where $I_{0}$ denotes the zeroth-order modified Bessel function.

\subsection{Implementation}

In this subsection, the concrete representations of the collision operator implemented in GKV code are shown. The term for collisions in Eq. (20) consists of the fieldparticle part and the test-particle part as follows:

$$
C_{a}^{(\mathrm{GK})}=\sum_{b} C_{a b}^{(\mathrm{GK})}=\sum_{b}\left(C_{a b}^{\mathrm{T}(\mathrm{GK})}+C_{a b}^{\mathrm{F}(\mathrm{GK})}\right) .
$$

Using the representations

$$
C_{a b}^{\mathrm{T}}\left(F_{a \mathrm{M}} v_{\|}\right)=-\theta_{a b} \frac{F_{a \mathrm{M}}}{\tau_{a b}}\left(1+\alpha_{a b}^{2}\right) v_{\|}
$$




$$
\begin{aligned}
& \times\left(\frac{3 \sqrt{\pi}}{2} \frac{G\left(\alpha_{a b} x_{a}\right)}{x_{a}}+\left(\theta_{a b}-1\right) \frac{\alpha_{a b}}{\left(1+\alpha_{a b}^{2}\right)^{3 / 2}}\right), \\
& C_{a b}^{\mathrm{T}}\left(F_{a \mathrm{M}} x_{a}^{2}\right)=-\theta_{a b} \frac{F_{a \mathrm{M}}}{\tau_{a b}}\left[\left(\theta_{a b}-1\right) \frac{2 \alpha_{a b}}{\left(1+\alpha_{a b}^{2}\right)^{3 / 2}}\left(x_{a}^{2}-\frac{3}{2}\right)\right. \\
& \left.+\frac{3 \sqrt{\pi}}{2 x_{a} \alpha_{a b}^{2}}\left(H\left(\alpha_{a b} x_{a}\right)-\alpha_{a b} x_{a} H^{\prime}\left(\alpha_{a b} x_{a}\right)\left(1+\alpha_{a b}^{2}\right)\right)\right]
\end{aligned}
$$

the field-particle part of the operator in Eqs. (11) or (22) can be expressed as

$$
\begin{aligned}
& C_{a b}^{\mathrm{F}(\mathrm{GK})}=-F_{a \mathrm{M}} \frac{\theta_{a b} \theta_{b a}}{\tau_{a b} \tau_{b a}}\left[\frac { m _ { a } m _ { b } } { \gamma _ { a b } T _ { a } } \left(\frac{3 \sqrt{\pi}}{2}\left(1+\alpha_{a b}^{2}\right) \frac{G\left(\alpha_{a b} x_{a}\right)}{x_{a}}\right.\right. \\
& \left.+\left(\theta_{a b}-1\right) \frac{\alpha_{a b}}{\left(1+\alpha_{a b}^{2}\right)^{1 / 2}}\right) \\
& \times\left\{J_{0}^{a} v_{\|}\left(\frac{3 \sqrt{\pi}}{2}\left(1+\alpha_{b a}^{2}\right) \mathcal{N}_{b a}^{(1)}+\left(\theta_{b a}-1\right) \frac{\alpha_{b a}}{\left(1+\alpha_{b a}^{2}\right)^{1 / 2}} \mathcal{N}_{b}^{(4)}\right)\right. \\
& \left.+J_{1}^{a} v_{\perp}\left(\frac{3 \sqrt{\pi}}{2}\left(1+\alpha_{b a}^{2}\right) \mathcal{N}_{b a}^{(6)}+\left(\theta_{b a}-1\right) \frac{\alpha_{b a}}{\left(1+\alpha_{b a}^{2}\right)^{1 / 2}} \mathcal{N}_{b}^{(7)}\right)\right\} \\
& +J_{0}^{a} \frac{T_{b}}{\eta_{a b}}\left\{\frac{3 \sqrt{\pi}}{2} \alpha_{a b}^{-2}\left[\frac{H\left(\alpha_{a b} x_{a}\right)}{x_{a}}-\alpha_{a b}\left(1+\alpha_{a b}^{2}\right) H^{\prime}\left(\alpha_{a b} x_{a}\right)\right]\right. \\
& \left.+\frac{\alpha_{a b}}{\left(1+\alpha_{a b}^{2}\right)^{3 / 2}}\left(\theta_{a b}-1\right)\left(x_{a}^{2}-\frac{3}{2}\right)\right\} \\
& \times\left\{\frac{3 \sqrt{\pi}}{2} \alpha_{b a}^{-2}\left[\mathcal{N}_{b a}^{(2)}-\alpha_{b a}\left(1+\alpha_{b a}^{2}\right) \mathcal{N}_{b a}^{(3)}\right]\right. \\
& \left.\left.+\frac{2 \alpha_{b a}}{\left(1+\alpha_{b a}^{2}\right)^{3 / 2}}\left(\theta_{b a}-1\right) \mathcal{N}_{b}^{(5)}\right\}\right] .
\end{aligned}
$$

Similarly, using the representations

$$
\begin{aligned}
& C_{a b}^{\mathrm{T} 0}\left(F_{a \mathrm{M}} v_{\|}\right)=-\frac{3 \sqrt{\pi}}{2 x_{a}} \frac{F_{a \mathrm{M}}}{\tau_{a b}}\left(1+\alpha_{a b}^{2}\right) v_{\|} G\left(\alpha_{a b} x_{a}\right), \\
& C_{a b}^{\mathrm{T} 0}\left(F_{a \mathrm{M}} x_{a}^{2}\right) \\
& =-\frac{3 \sqrt{\pi}}{2 x_{a} \alpha_{a b}^{2}} \frac{F_{a \mathrm{M}}}{\tau_{a b}}\left(H\left(\alpha_{a b} x_{a}\right)-\alpha_{a b} x_{a} H^{\prime}\left(\alpha_{a b} x_{a}\right)\left(1+\alpha_{a b}^{2}\right)\right),
\end{aligned}
$$

the test-particle part of the collision operator in Eqs. (6) or (22) can be represented as

$$
C_{a b}^{\mathrm{T}(\mathrm{GK})}=C_{a b}^{\mathrm{V}(\mathrm{GK})}+C_{a b}^{\mathrm{D}(\mathrm{GK})},
$$

where $C_{a b}^{\mathrm{D}(\mathrm{GK})}$ is the different temperature part,

$$
\begin{aligned}
& C_{a b}^{\mathrm{D}(\mathrm{GK})}=-\left(\theta_{a b}-1\right) \frac{3 \sqrt{\pi}}{2} \frac{F_{a \mathrm{M}}}{n_{a}} \tau_{a b}^{-1} \\
& \times\left[\frac{m_{a}}{T_{a}}\left(1+\alpha_{a b}^{2}\right)\left(J_{0}^{a} v_{\|} \mathcal{N}_{a b}^{(1)}+J_{1}^{a} v_{\perp} \mathcal{N}_{a b}^{(6)}\right)\right. \\
& \left.+\frac{2}{3} J_{0}^{a}\left(x_{a}^{2}-\frac{3}{2}\right) \alpha_{a b}^{-2}\left(\mathcal{N}_{a b}^{(2)}-\alpha_{a b}\left(1+\alpha_{a b}^{2}\right) \mathcal{N}_{a b}^{(3)}\right)\right] \\
& -\left(\theta_{a b}-1\right) \frac{3 \sqrt{\pi}}{2} \frac{F_{a \mathrm{M}}}{n_{a}} \tau_{a b}^{-1} x_{a}^{-1}\left[J_{0}^{a} v_{\|} \frac{m_{a}}{T_{a}}\left(1+\alpha_{a b}^{2}\right) G\left(\alpha_{a b} x_{a}\right) \mathcal{N}_{a}^{(4)}\right. \\
& +J_{1}^{a} v_{\perp} \frac{m_{a}}{T_{a}}\left(1+\alpha_{a b}^{2}\right) G\left(\alpha_{a b} x_{a}\right) \mathcal{N}_{a}^{(7)} \\
& \left.+\frac{2}{3} J_{0}^{a} \alpha_{a b}^{-2}\left[H\left(\alpha_{a b} x_{a}\right)-\alpha_{a b} x_{a}\left(1+\alpha_{a b}^{2}\right) H^{\prime}\left(\alpha_{a b} x_{a}\right)\right] \mathcal{N}_{a}^{(5)}\right]
\end{aligned}
$$

$-\left(\theta_{a b}-1\right)^{2} \frac{F_{a \mathrm{M}}}{n_{a}} \tau_{a b}^{-1} \frac{\alpha_{a b}}{\left(1+\alpha_{a b}^{2}\right)^{1 / 2}}\left[J_{0}^{a} v_{\|} \frac{m_{a}}{T_{a}} \mathcal{N}_{a}^{(4)}+J_{1}^{a} v_{\perp} \frac{m_{a}}{T_{a}} \mathcal{N}_{a}^{(7)}\right.$$$
\left.+\frac{2}{1+\alpha_{a b}^{2}} J_{0}^{a}\left(x_{a}^{2}-\frac{3}{2}\right) \mathcal{N}_{a}^{(5)}\right]
$$

and $C_{a b}^{\mathrm{V}(\mathrm{GK})}$ is given by

$$
\begin{aligned}
C_{a b}^{\mathrm{V}(\mathrm{GK})}= & \left(2 v_{0}^{a b}(v) x_{a}^{2}-\frac{k_{\perp}^{2}}{4 \Omega_{a}^{2}} v_{\mathrm{D}}^{a b}(v)\left(2 v_{\|}^{2}+v_{\perp}^{2}\right)+v_{\|}^{a b}(v) v_{\perp}^{2}\right) h_{k_{\perp}}^{a} \\
& +v_{\|}^{a b}(v) x_{a}^{2}\left(1-\alpha_{a b}^{2}\right) v_{\|} \frac{\partial}{\partial v_{\|}} h_{k_{\perp}}^{a} \\
& +\left(v_{\|}^{a b}(v) x_{a}^{2}\left(1-\alpha_{a b}^{2}\right)+\frac{1}{2} v_{\mathrm{D}}^{a b}(v)\left(\frac{v_{\|}^{2}}{v_{\perp}^{2}}+1\right)\right) v_{\perp} \frac{\partial}{\partial v_{\perp}} h_{k_{\perp}}^{a} \\
& +\left(v_{\|}^{a b}(v)-v_{\mathrm{D}}^{a b}(v)\right) v_{\|} v_{\perp} \frac{\partial^{2}}{\partial v_{\|} \partial v_{\perp}} h_{k_{\perp}}^{a} \\
& +\frac{1}{2}\left(v_{\|}^{a b}(v) v_{\perp}^{2}+v_{\mathrm{D}}^{a b} v_{\|}^{2}\right) \frac{\partial^{2}}{\partial v_{\perp}^{2}} h_{k_{\perp}}^{a} \\
& +\frac{1}{2}\left(v_{\|}^{a b}(v) v_{\|}^{2}+v_{\mathrm{D}}^{a b}(v) v_{\perp}^{2}\right) \frac{\partial^{2}}{\partial v_{\|}^{2}} h_{k_{\perp}}^{a} .
\end{aligned}
$$

Here, we define $v_{0}^{a b}(v) \equiv(3 \sqrt{\pi} / 4) \alpha_{a b} \tau_{a b}^{-1} x_{a}^{-2} H^{\prime}\left(x_{b}\right)$. In the developed code, we employ fourth-order finite difference schemes in the velocity space derivatives, and the velocity moments of the variables employed in the above expressions are given by

$$
\begin{aligned}
\mathcal{N}_{a b}^{(1)} & \equiv \int d^{3} v J_{0}^{a} h_{k_{\perp}}^{a} v_{\|} \frac{G\left(\alpha_{a b} x_{a}\right)}{x_{a}}, \\
\mathcal{N}_{a b}^{(2)} & \equiv \int d^{3} v J_{0}^{a} h_{k_{\perp}}^{a} \frac{H\left(\alpha_{a b} x_{a}\right)}{x_{a}}, \\
\mathcal{N}_{a b}^{(3)} & \equiv \int d^{3} v J_{0}^{a} h_{k_{\perp}}^{a} H^{\prime}\left(\alpha_{a b} x_{a}\right), \\
\mathcal{N}_{a}^{(4)} & \equiv \int d^{3} v J_{0}^{a} h_{k_{\perp}}^{a} v_{\|}, \\
\mathcal{N}_{a}^{(5)} & \equiv \int d^{3} v J_{0}^{a} h_{k_{\perp}}^{a}\left(x_{a}^{2}-\frac{3}{2}\right), \\
\mathcal{N}_{a b}^{(6)} & \equiv \int d^{3} v J_{1}^{a} h_{k_{\perp}}^{a} v_{\perp} \frac{G\left(\alpha_{a b} x_{a}\right)}{x_{a}}, \\
\mathcal{N}_{a}^{(7)} & \equiv \int d^{3} v J_{1}^{a} h_{k_{\perp}}^{a} v_{\perp} .
\end{aligned}
$$

\section{Numerical Tests}

To validate the developed collision operator, we perform the calculations for the Maxwellian relaxation process, the thermal equilibration, and the collisional damping of the zonal flow potentials, and also evaluate numerical errors found in the conservation properties and the selfadjointness relations discussed in Sec.2. For simplicity, we consider the drift kinetic limit of the model collision operator $\left(k_{\perp} \rho_{\mathrm{i}} \ll 1\right)$ except for the zonal flow damping test in Sec. 4.3, as the polarization effect is influential in the zonal-flow damping.

\subsection{Maxwellian relaxation}

The relaxation processes with the test-particle colli- 
sion operator can be described by

$$
\frac{\partial}{\partial t} \delta f_{a}=C_{a b}^{\mathrm{T}}\left(\delta f_{a}\right)
$$

If the test-particle operator forms the Lenard-Berstein type [17], which is employed in previous GKV simulations,

$$
\begin{aligned}
& C_{a b}^{\mathrm{T}}\left(\delta f_{a}\right)=C^{(\mathrm{LB})}\left(\delta f_{a}\right) \\
& =v_{a}\left[\frac{1}{v_{\perp}} \frac{\partial}{\partial v_{\perp}}\left(v_{\perp} \frac{\partial}{\partial v_{\perp}} \delta f_{a}+\frac{v_{\perp}^{2}}{v_{\mathrm{t} a}^{2}} \delta f_{a}\right)\right. \\
& \left.+\frac{\partial}{\partial v_{\|}}\left(\frac{\partial}{\partial v_{\|}} \delta f_{a}+\frac{v_{\|}}{v_{\mathrm{t} a}^{2}} \delta f_{a}\right)\right],
\end{aligned}
$$

since the collision frequency in the operator is independent of velocity, the distribution function uniformly evolves to the Maxwellian form in the velocity space from its initial distribution. Here, $v_{a}$ denotes the collision frequency, and $v_{\mathrm{t} a}=\left(T_{a} / m_{a}\right)^{1 / 2}$ is the thermal velocity of the ion. Now we consider the initial distribution function as a bell-shape function,

$$
\delta f_{a}(t=0)=\exp \left[-\left(\frac{v}{3 v_{\mathrm{t} a}}\right)^{4}\right] .
$$

Time evolution of the distribution function by the ionion collisional relaxation process due to Eq. (38) with the Lenard-Berstein form in Eq. (39) is shown in Fig. 1-(a) for the case of $v_{a}=0.0076\left(v_{\mathrm{ti}} / R_{0}\right)$, where the distribution goes to the Maxwellian uniformly in the velocity space. Here, $R_{0}$ is the major radius. Figure 1-(b) shows the time evolution of the distribution function by the test-particle operator for ion-ion collisions given by Eq. (28) in the drift-kinetic limit with equal temperatures and $v_{\mathrm{ii}}=\tau_{\mathrm{ii}}^{-1}=0.038\left(v_{\mathrm{ti}} / R_{0}\right)$. The frequency of the operator has the velocity-dependence, so that it becomes higher for smaller velocity but lower for larger velocity. Therefore, in the figure, we can see that the distribution function relaxes to the Maxwellian faster in the small $\left|v_{\|}\right|$region than in the large $\left|v_{\|}\right|$region.

\subsection{Thermal equilibration}

In this section, we conduct a test of thermal equilibration processes with the model collision operator in a threeion species plasma. Here, we consider the multi-species plasma consists of deuterium (D), helium (He) and carbon (C). If each ion species has the perturbed Maxwellian distributions with different background temperature and its fluctuations, we have

$$
\delta f_{a}=F_{a \mathrm{M}}\left(\frac{\delta n_{a}}{n_{a}}+\frac{m_{a}}{T_{a}} \boldsymbol{u}_{a} \cdot \boldsymbol{v}+\frac{\delta T_{a}}{T_{a}}\left(\frac{m_{a} v^{2}}{2 T_{a}}-\frac{3}{2}\right)\right),
$$

where $\boldsymbol{u}_{a}$ is the mean flow. Thermal equilibration among the ion species proceeds as described by the following equation:

$$
\frac{\partial}{\partial t} \delta f_{a}=\sum_{b}\left(C_{a b}^{\mathrm{T}(\mathrm{DK})}\left(\delta f_{a}\right)+C_{a b}^{\mathrm{F}(\mathrm{DK})}\left(\delta f_{b}\right)\right),
$$
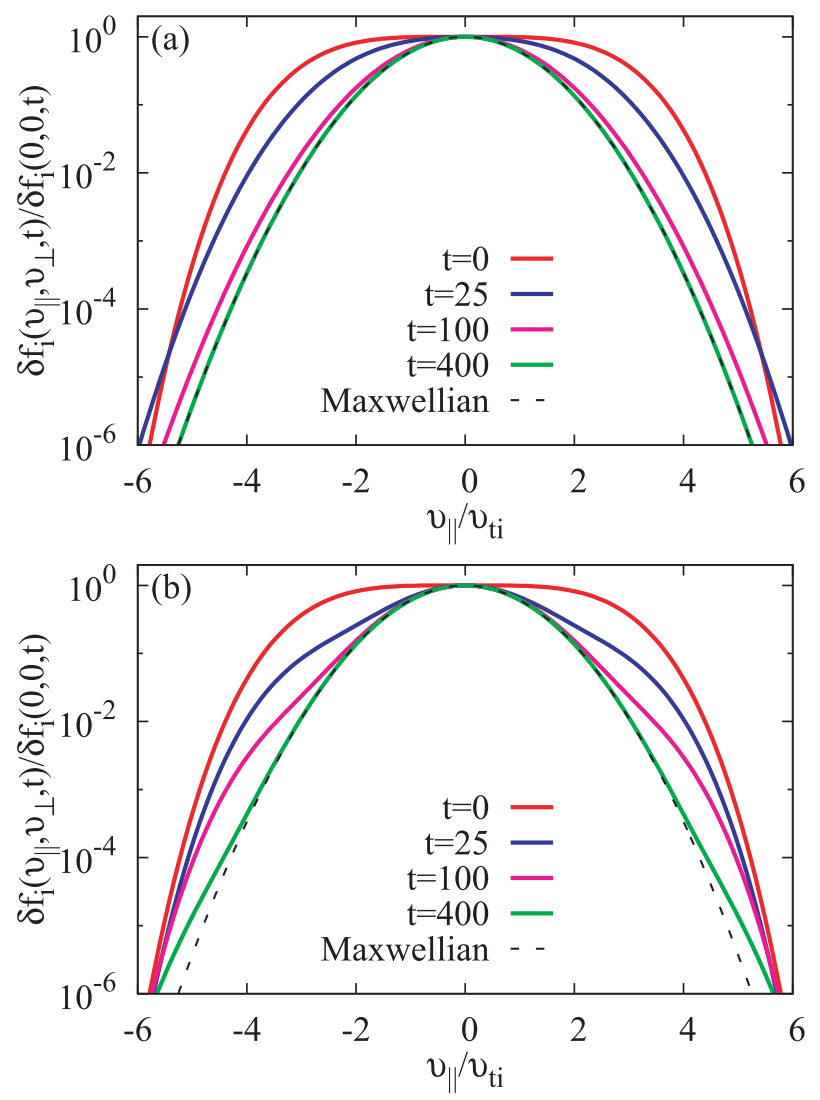

Fig. 1 Time evolutions of the distribution function under the collisional relaxation processes, obtained by different testparticle collision operators; (a) the Lenard-Berstein operator with $v_{a} /\left(v_{\mathrm{ti}} / R_{0}\right)=0.0076$ and (b) the model collision operator Eq. (28) with $v_{\mathrm{ii}} /\left(v_{\mathrm{ti}} / R_{0}\right)=0.038$ for the same species ion-ion collision. The plots are obtained in $v_{\perp}=0$.

where the temperature fluctuations and the mean flow relax to $\delta T_{a} / T_{a}=\delta T_{b} / T_{b}$ and $\boldsymbol{u}_{a}=\boldsymbol{u}_{b}$, respectively. Figure 2 plots the time evolutions of the temperature fluctuations $\delta T_{a} / T_{a}$ during the equilibration process, where the initial distributions are given by Eq. (41) with $\boldsymbol{u}_{a}=0, T_{\mathrm{He}} / T_{\mathrm{D}}=$ 1.2, and $T_{\mathrm{C}} / T_{\mathrm{D}}=0.8$. The initial charge densities are given by $Z_{\mathrm{D}} n_{\mathrm{D}}: Z_{\mathrm{He}} n_{\mathrm{He}}: Z_{\mathrm{C}} n_{\mathrm{C}}=0.20: 0.75: 0.05$. We observe that $\delta T_{a} / T_{a}$ converges under the thermal relaxation imposed by the implemented collision operator. For this calculation, $\left(N_{v_{\|}}, N_{v_{\perp}}\right)=(96,48)$ grid points in $\left(v_{\|}, v_{\perp}\right)$ space with $v_{\max }=6 v_{\mathrm{t} a}$, which are used in typical GKV simulations, are employed. The thermal equilibration process proceeds with the heat exchange between multiple species with different temperatures, the distribution functions converge to the same form after a sufficiently long time. According to the H-theorem, Eq. (19), the entropy are monotonically produced due to the collisional heat exchange. The distribution functions at $t /\left(R_{0} / v_{\mathrm{tp}}\right)=0$ and $t /\left(R_{0} / v_{\text {tp }}\right)=2000$ are plotted in Fig. 3 , where it is clearly seen that each distribution function goes to the same form. Here, $v_{\mathrm{tp}}$ is the thermal velocity of proton. 


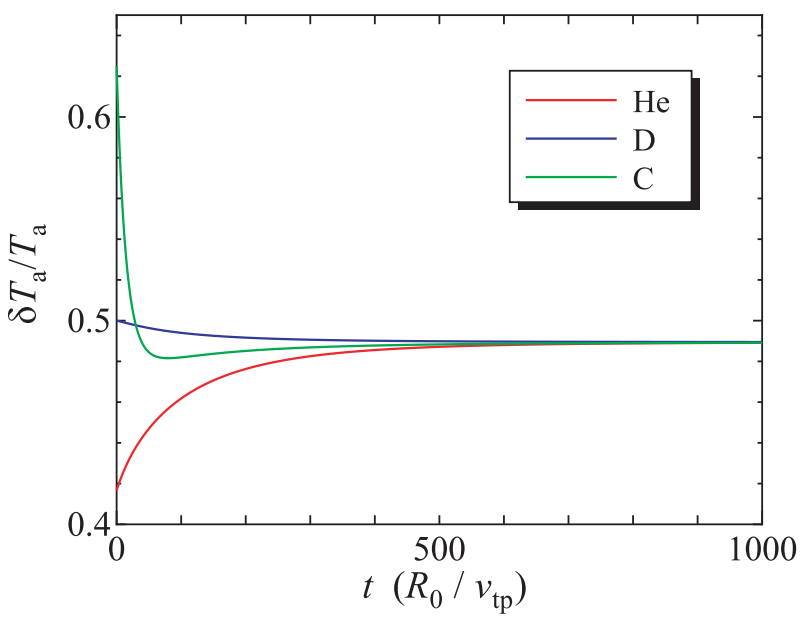

Fig. 2 Time evolutions of the temperature fluctuations $\delta T_{a} / T_{a}$ of Helium ( $a=\mathrm{He}$; red curve), Deuterium ( $a=\mathrm{D}$; blue), and Carbon ( $a=\mathrm{C}$; green).

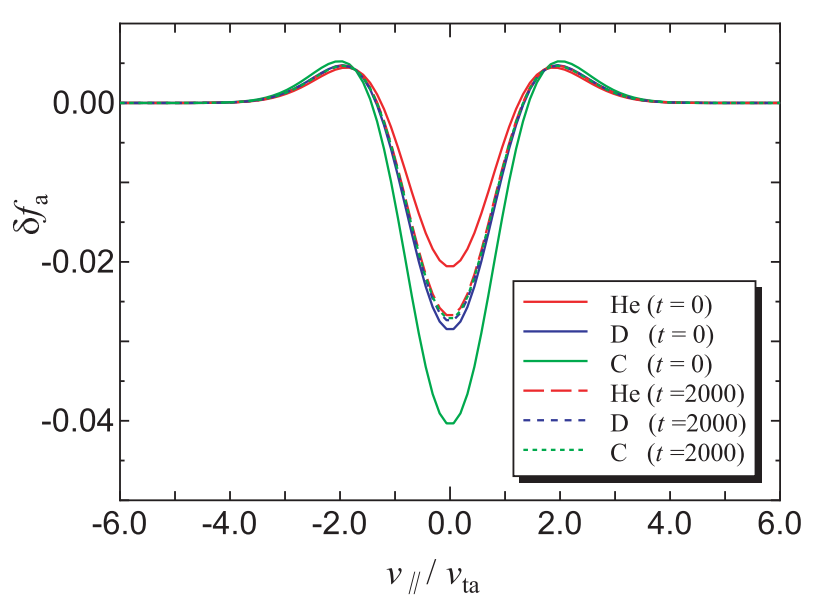

Fig. 3 Distribution functions $\delta f_{a}$ on $v_{\perp}=0$ at $t /\left(R_{0} / v_{\text {tp }}\right)=0$ (solid curves) and $t /\left(R_{0} / v_{\mathrm{tp}}\right)=2000$ (dashed curves). The red curves represent the results for $a=\mathrm{He}$, the blue is $a=\mathrm{D}$, and the green is $a=\mathrm{C}$.

\subsection{Zonal flow evolution}

As an application of the developed collision operator to the gyrokinetic calculations, we evaluate the collisional linear response of the zonal flow potential. The zonal flows are given by an electrostatic potential perturbation that varies in the radial direction but remains constant on the flux surface. Hence, the zonal flow component $\left\langle\delta \phi_{k_{\perp}}\right\rangle_{\mathrm{ZF}}$ in Eq. (21) is evaluated at $k_{y}=0$, where $k_{y}$ denotes the perpendicular wavenumber along the poloidal direction. In the tests, we calculate time evolutions of the zonal flow potential in the linear response to the Maxwellian initial perturbation solving the linear gyrokinetic equation based on Eq. (20) with the gyrokinetic collision operator for singleion species. Here, we assume the electron density perturbation to be given by $\delta n_{e}=\left(n_{0} e / T_{e}\right)\left(\delta \phi_{k_{\perp}}-\left\langle\delta \phi_{k_{\perp}}\right\rangle_{\mathrm{ZF}}\right)$, and we employ the Cyclone base case configuration [18] with the parameters of $\varepsilon_{\mathrm{t}}=r / R_{0}=0.18$ and $q=1.42$. Figure 4

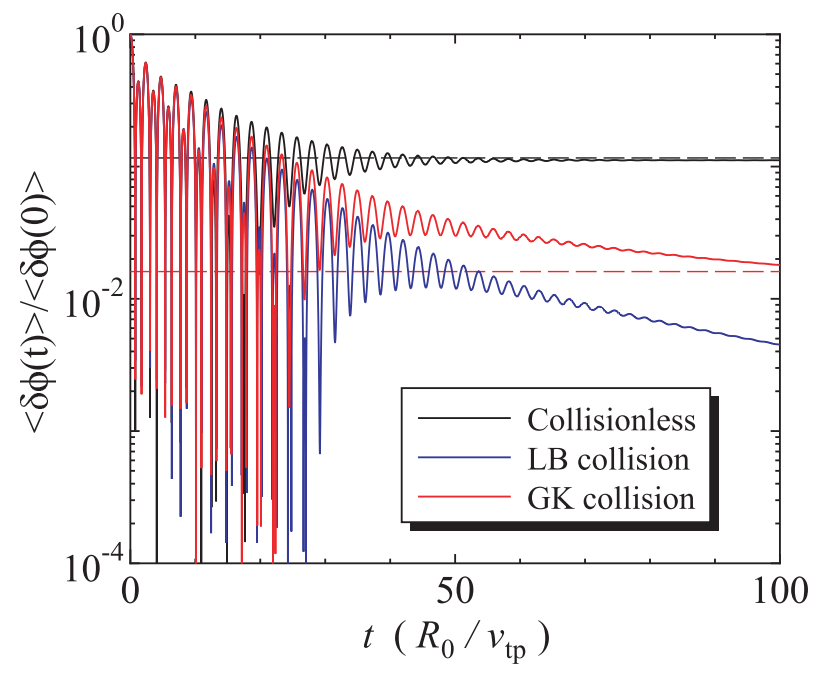

Fig. 4 Collisional linear responses of the zonal flow potentials of the single ion species plasma with the developed gyrokinetic collision operator (red curve) and the LenardBernstein operator (blue curve) with $v_{\mathrm{i}}=(3 \sqrt{\pi} / 4) \tau_{\mathrm{ii}}^{-1}$. The black curve shows the response in collisionless case. The horizontal dashed lines are the long-time analytic limits in the collisionless case (black) Eq. (43) and the collisional case (red) Eq. (44). The plots are obtained in $k_{x} \rho_{\mathrm{tp}}=0.1$.

plots the linear responses of the zonal flow potential with the radial wavenumber of $k_{x} \rho_{\text {tp }}=0.1$. The results are compared between the developed gyrokinetic collision operator and the Lenard-Bernstein model with $v_{a}=(3 \sqrt{\pi} / 4) \tau_{a a}^{-1}$. For the calculations, we used $\left(N_{v_{\|}}, N_{v_{\perp}}\right)=(256,64)$ grid points in the velocity space with $v_{\max }=5 v_{\mathrm{t} a}$. As seen in the plots, the response functions of the zonal flow potential with the two operators oscillate similarly in the initial phase $\left(t /\left(R_{0} / v_{\text {tp }}\right)<20\right)$, but deviate after a long time $\left(t /\left(R_{0} / v_{\mathrm{tp}}\right)>20\right)$. Here, we note that the long-time limit of the response function has been analytically obtained as

$$
\frac{\delta \phi_{k_{\perp}}(t)}{\delta \phi_{k_{\perp}}(0)} \rightarrow \frac{1}{1+1.6 q^{2} / \varepsilon_{\mathrm{t}}^{1 / 2}},
$$

in the collisionless case [19]. On the other hand, in the collisional case, the potential approaches the value,

$$
\frac{\delta \phi_{k_{\perp}}(t)}{\delta \phi_{k_{\perp}}(0)} \rightarrow \frac{\varepsilon_{\mathrm{t}}^{2}}{q^{2}},
$$

where the toroidal momentum conservation leads to that steady state value [20]. In Fig.4, we can see that the residual potential in the developed collision operator case approaches the finite value close to the analytic prediction Eq. (44), while the Lenard-Bernstein model leads to the over-damping. The developed collision operator also yields the different collisionality from the LenardBernstein model by virtue of Eq. (22). Therefore, it is considered that the differences found in Fig. 4 are caused by the fact that the velocity-dependent collisionality and fieldparticle part are included in the developed operator with more accurate momentum conservation. 
In addition, we consider the multi-species plasma consists of deuterium (D), helium (He) and carbon (C), with the initial conditions as same as Sec.4.2. Figure 5 shows the zonal flow response functions calculated with the developed collision operator for the single- and multi-species cases. The zonal flow damping in the multi-species case is also tested by using the developed collision operator in the drift kinetic limit. In the plots, one finds the geodesic acoustic mode (GAM) frequency is lowered by increase of the effective $Z$ number in the multi-species ion case. For the longer-time response of zonal flows, a certain difference between the gyrokinetic and drift kinetic cases appears although the difference is small since we adopted a small perpendicular wavenumber, i.e., $k_{\perp} \rho_{\text {tp }}=0.1$, in the calculation. The above results suggest that components of the multi-species plasmas may influence the turbulent transport phenomena by changing the collisionality and the zonal flow response function.

\subsection{Error estimation}

In order to estimate numerical errors in calculations with the developed collision operator, we should consider the acceptable level of the errors in the local gyrokinetic simulations. Typical GKV simulations require a calculation duration time of $t_{\mathrm{sim}} \sim 10^{2}\left(R_{0} / v_{\mathrm{ti}}\right)$ to reach the turbulent transport saturation. Therefore, if we request an acceptable error $\Delta_{\lim }$ of less than $1 \%$ over the whole duration, the cumulative error in the calculation with the collision term $t_{\text {sim }}\left|\Delta_{\text {lim }}\right|$ should not exceed $10^{-2}$, i.e, we specify $\left|\Delta_{\text {lim }}\right|<10^{-4}$ as the upper-limit error. In the follow-

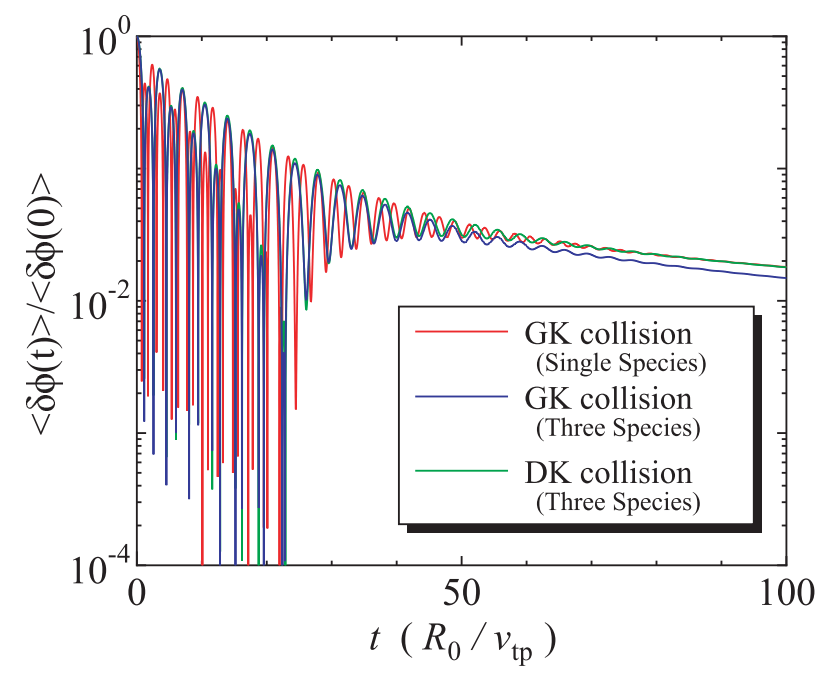

Fig. 5 Collisional linear responses of the zonal flow potentials of the three ion species plasmas with the developed gyrokinetic collision operator (blue curve). The red curve shows the result in the case of the single ion species shown in Fig. 4, and the green curve shows the result in the case of the three ion species plasmas with the developed operator in the drift kinetic limit. The plots are obtained in $k_{x} \rho_{\text {tp }}=0.1$. ing tests, the grid points and box size of the velocity space were those specified in Sec. 4.2.

\subsection{1 adjointness relations}

In order to estimate the errors of the developed collision operator for the adjointness relations in Eqs. (17) and (18), we define the relative errors as follows:

$$
\begin{aligned}
& \Delta_{a b}^{(\text {adj)T }} \equiv \tau_{a b}\left(\int d^{3} v \frac{h_{a}}{F_{a \mathrm{M}}} C_{a b}^{\mathrm{T}}\left(g_{b}\right)-\int d^{3} v \frac{g_{b}}{F_{a \mathrm{M}}} C_{a b}^{\mathrm{T}}\left(h_{a}\right)\right) \\
& /\left(\int d^{3} v \frac{h_{a}^{2}}{F_{a \mathrm{M}}} \int d^{3} v \frac{g_{b}^{2}}{F_{a \mathrm{M}}}\right)^{1 / 2},
\end{aligned}
$$

for the test particle part, and

$$
\begin{aligned}
& \Delta_{a b}^{(\mathrm{adj}) \mathrm{F}} \equiv \tau_{a b}\left(T_{a} \int d^{3} v \frac{h_{a}}{F_{a \mathrm{M}}} C_{a b}^{\mathrm{F}}\left(g_{b}\right)-T_{b} \int d^{3} v \frac{g_{b}}{F_{b \mathrm{M}}} C_{b a}^{\mathrm{F}}\left(h_{a}\right)\right) \\
& /\left(\int d^{3} v \frac{h_{a}^{2}}{F_{a \mathrm{M}}} \int d^{3} v \frac{g_{b}^{2}}{F_{b \mathrm{M}}}\right)^{1 / 2},
\end{aligned}
$$

for the field particle part. As the test distribution functions, we employ the perturbed Maxwellian forms as follows:

$$
\begin{aligned}
& h_{a}=F_{a \mathrm{M}}\left(\frac{\delta n_{a}}{n_{a}}+\frac{m_{a}}{T_{a}} u_{a} v_{\|}+\frac{\delta T_{a}}{T_{a}}\left(\frac{m_{a} v^{2}}{2 T_{a}}-\frac{3}{2}\right)\right), \\
& g_{b}=F_{b \mathrm{M}}\left(\frac{\delta n_{b}}{n_{b}}+\frac{m_{b}}{T_{b}} u_{b} v_{\|}+\frac{\delta T_{b}}{T_{b}}\left(\frac{m_{b} v^{2}}{2 T_{b}}-\frac{3}{2}\right)\right),
\end{aligned}
$$

with the normalizations of the zero-th order moments,

$$
\int d^{3} v h=1, \text { and } \int d^{3} v g=1 .
$$

The error about the H-theorem is written as the negative definition,

$$
\begin{aligned}
\Delta_{a b}^{\mathrm{H}} & =T_{a} \int d^{3} v \frac{h_{a}}{F_{a \mathrm{M}}}\left(C_{a b}^{\mathrm{T}(\mathrm{GK})}\left(h_{a}\right)+C_{a b}^{\mathrm{F}(\mathrm{GK})}\left(g_{b}\right)\right) \\
& +T_{b} \int d^{3} v \frac{h_{b}}{F_{b \mathrm{M}}}\left(C_{b a}^{\mathrm{T}(\mathrm{GK})}\left(g_{b}\right)+C_{b a}^{\mathrm{F}(\mathrm{GK})}\left(h_{a}\right)\right) \leq 0 .
\end{aligned}
$$

The calculated errors are summarized in Table 1. All the

Table 1 Relative errors of the collision operator for the selfadjointness in multi-species plasmas with helium (He), deuterium (D), and carbon (C).

\begin{tabular}{c||c|c|c}
$a b$ & $\Delta_{a b}^{(\mathrm{adj}) \mathrm{T}}$ & $\Delta_{a b}^{(\mathrm{adj}) \mathrm{F}}$ & $\Delta_{a b}^{\mathrm{H}}$ \\
\hline \hline $\mathrm{He} \mathrm{He}$ & $-3.10 \times 10^{-8}$ & $-1.15 \times 10^{-17}$ & $-5.47 \times 10^{-6}$ \\
$\mathrm{He} \mathrm{D}$ & $-6.24 \times 10^{-7}$ & $3.28 \times 10^{-16}$ & $-1.10 \times 10^{-5}$ \\
$\mathrm{He} \mathrm{C}$ & $1.82 \times 10^{-6}$ & $5.52 \times 10^{-17}$ & $-7.56 \times 10^{-6}$ \\
$\mathrm{D} \mathrm{He}$ & $9.05 \times 10^{-7}$ & $-5.50 \times 10^{-16}$ & $-1.84 \times 10^{-5}$ \\
$\mathrm{D} \mathrm{D}$ & $-3.10 \times 10^{-8}$ & $1.58 \times 10^{-15}$ & $-3.12 \times 10^{-5}$ \\
$\mathrm{D} \mathrm{C}$ & $2.09 \times 10^{-6}$ & $9.86 \times 10^{-17}$ & $-2.50 \times 10^{-5}$ \\
$\mathrm{C} \mathrm{He}$ & $-1.63 \times 10^{-7}$ & $5.90 \times 10^{-18}$ & $-3.02 \times 10^{-6}$ \\
$\mathrm{C} \mathrm{D}$ & $2.81 \times 10^{-7}$ & $4.12 \times 10^{-18}$ & $-1.03 \times 10^{-5}$ \\
$\mathrm{C} \mathrm{C}$ & $-3.10 \times 10^{-8}$ & $-1.25 \times 10^{-17}$ & $-1.83 \times 10^{-6}$
\end{tabular}


Table 2 Relative errors of the collision operator for the conservations of particle, momentum, and energy in multi-species plasmas with deuterium, helium and carbon.

\begin{tabular}{c||c|c|c|c}
$a b$ & $\Delta_{a b}^{(0) \mathrm{T}}$ & $\Delta_{a b}^{(0) \mathrm{F}}$ & $\Delta_{a b}^{(1)}$ & $\Delta_{a b}^{(2)}$ \\
\hline \hline $\mathrm{He} \mathrm{He}$ & $-4.13 \times 10^{-5}$ & $1.63 \times 10^{-6}$ & $7.27 \times 10^{-6}$ & $-1.57 \times 10^{-5}$ \\
$\mathrm{He} \mathrm{D}$ & $-2.99 \times 10^{-5}$ & $4.93 \times 10^{-7}$ & $4.71 \times 10^{-6}$ & $-2.34 \times 10^{-5}$ \\
$\mathrm{He} \mathrm{C}$ & $-6.53 \times 10^{-5}$ & $1.44 \times 10^{-5}$ & $8.62 \times 10^{-6}$ & $-9.28 \times 10^{-6}$ \\
D He & $-5.75 \times 10^{-5}$ & $3.24 \times 10^{-6}$ & $3.17 \times 10^{-5}$ & $-4.64 \times 10^{-5}$ \\
D D & $-4.13 \times 10^{-5}$ & $1.63 \times 10^{-6}$ & $2.73 \times 10^{-5}$ & $-5.88 \times 10^{-5}$ \\
D C & $-6.28 \times 10^{-5}$ & $3.23 \times 10^{-5}$ & $3.45 \times 10^{-5}$ & $-3.92 \times 10^{-5}$ \\
C He & $-3.66 \times 10^{-5}$ & $2.21 \times 10^{-8}$ & $8.18 \times 10^{-9}$ & $-1.43 \times 10^{-5}$ \\
C D & $-4.47 \times 10^{-5}$ & $-6.85 \times 10^{-7}$ & $-3.30 \times 10^{-7}$ & $-1.86 \times 10^{-5}$ \\
C C & $-4.13 \times 10^{-5}$ & $1.63 \times 10^{-6}$ & $1.82 \times 10^{-6}$ & $-3.92 \times 10^{-6}$
\end{tabular}

errors are within the acceptable level of the local gyrokinetic simulations. Regarding the $\mathrm{H}$-theorem, it is seen that the all values of $\Delta^{\mathrm{H}}$ are negative.

\subsection{2 conservation properties}

Based on the conservation laws for particle [Eq. (14)], momentum [Eq.(15)], and energy [Eq.(16)], we also check the errors defined as

$$
\begin{aligned}
& \Delta_{a b}^{(0) \mathrm{T}}=\tau_{a b} \int d^{3} v C_{a b}^{\mathrm{T}(\mathrm{DK})}\left(h_{a}\right) / \int d^{3} v h_{a}, \\
& \Delta_{a b}^{(0) \mathrm{F}}=\tau_{a b} \int d^{3} v C_{a b}^{\mathrm{F}(\mathrm{DK})}\left(h_{a}\right) / \int d^{3} v h_{a},
\end{aligned}
$$

for the particle conservation,

$$
\begin{aligned}
& \Delta_{a b}^{(1)}=\tau_{a b}\left(\int d^{3} v m_{a} v_{\|} C_{a b}^{\mathrm{T}(\mathrm{DK})}\left(h_{a}\right)\right. \\
& \left.+\int d^{3} v m_{b} v_{\|} C_{b a}^{\mathrm{F}(\mathrm{DK})}\left(h_{a}\right)\right) / \int d^{3} v m_{a} v_{\|} h_{a},
\end{aligned}
$$

for the momentum conservation, and

$$
\begin{aligned}
& \Delta_{a b}^{(2)}=\tau_{a b}\left(\int d^{3} v m_{a} v^{2} C_{a b}^{\mathrm{T}(\mathrm{DK})}\left(h_{a}\right)\right. \\
& \left.+\int d^{3} v m_{b} v^{2} C_{b a}^{\mathrm{F}(\mathrm{DK})}\left(h_{a}\right)\right) / \int d^{3} v m_{a} v^{2} h_{a},
\end{aligned}
$$

for the energy conservation between the species $a$ and $b$ with the test distribution function

$$
h_{a}=F_{a \mathrm{M}}\left(\frac{\delta n_{a}}{n_{a}}+\frac{m_{a}}{T_{a}} u_{a} v_{\|}+\frac{\delta T_{a}}{T_{a}}\left(\frac{m_{a} v^{2}}{2 T_{a}}-\frac{3}{2}\right)\right),
$$

where the zero-th order moment of the perturbed distribution function is normalized as

$$
\int d^{3} v h_{a}=1
$$

Table 2 summarizes the errors in the conservation properties. Again, all the errors are within the acceptable levels of the local gyrokinetic simulations.

\section{Summary}

In this paper, we reported implementation of a numerical collision operator developed for multiple ion species plasma to the local gyrokinetic flux-tube code, GKV. The developed operator satisfies the conservation laws of particles, momentum, and energy, as well as the adjointness relations even for collisions among different particle species. The developed operator works well in numerical tests of the relaxation process, the thermal equilibration, and the collisional zonal flow damping. It is also confirmed that the new operator satisfies the several conservation properties within the acceptable error levels in local gyrokinetic turbulence simulations. The newly implemented collision operator to the GKV code is sufficient for practical use in the turbulent transport analysis of fusion plasmas, while the numerical errors may be enhanced in the case of high collisionality with heavy impurity ions, or in a longer time simulations in case near the marginal stability influence. We have confirmed that the numerical errors of the new operator for the multi-species ions are within the acceptable levels at least for the grid points and the box size of the velocity space which are employed in the present GKV simulations. In the case of the multi-species plasmas including electrons, the convergences of the numerical schemes may be modified because the collision operator requires higher resolution in the velocity space than the only ion case. More detailed analysis for strong collisional case and the convergences of the numerical schemes will be pursued in future works.

\section{Acknowledgments}

This work is supported by the Japanese Ministry of Education, Culture, Sports, Science, and Technology, Grant Nos. 26820398 and 26820401 in part by the NIFS collaborative Research Programs (NIFS13KNST057, NIFS14KNTT024, NIFS14KNTT026, and NIFS14KNST065), and by use of Helios system at International Fusion Energy Research Center (Project code: VLDGK_ST, GTNAXIS, 
GKTBMDL, and MSGKV2). Part of the results is obtained by using the $\mathrm{K}$ computer at the RIKEN Advanced Institute for Computational Science (Proposal number hp140053).

[1] T.-H. Watanabe and H. Sugama, Phys. Plasmas 11, 1476 (2004).

[2] X.Q. Xu and M.N. Rosenbluth, Phys. Fluids B 3, 627 (1991).

[3] Z. Lin et al., Phys. Plasmas 2, 2975 (1995).

[4] W.X. Wang et al., Plasma Phys. Control. Fusion 41, 1091 (1999).

[5] M. Barnes et al., Phys. Plasmas 16, 072107 (2009).

[6] R.A. Kolesnikov et al., J. Comput. Phys. 229, 5564 (2010).

[7] S. Satake et al., Comput. Phys. Commun. 181, 1069 (2010).

[8] Y. Idomura et al., Plasma Fusion Res. 9, 3503028 (2014).

[9] H. Sugama, T.-H. Watanabe and M. Nunami, Phys. Plasmas 16, 112503 (2009).

[10] T.-H. Watanabe and H. Sugama, Nucl. Fusion 46, 24
(2006).

[11] M. Nunami, T.-H. Watanabe and H. Sugama, Plasma Fusion Res. 5, 016 (2010).

[12] P. Helander and D.J. Sigmer, Collisional Transport in Magnetized Plasmas (Cambridge University Press, Cambridge, 2002) Chap. 3.

[13] M. Nunami, T.-H. Watanabe and H. Sugama, Phys. Plasmas 19, 042504 (2012).

[14] A. Komori et al., Fusion Sci. Technol. 58, 1 (2010).

[15] K. Tanaka et al., Plasma Fusion Res. 5, S2053 (2010).

[16] A. Ishizawa et al., 25th IAEA Fusion Energy Conference, TH/P6-40 (2014).

[17] A. Lenard and I.B. Bernstein, Phys. Rev. 112, 1456 (1958).

[18] A.M. Dimits et al., Phys. Plasmas 7, 969 (2000).

[19] M.N. Rosenbluth and F.L. Hinton, Phys. Rev. Lett. 80, 724 (1998).

[20] F.L. Hinton and M.N. Rosenbluth, Plasma Phys. Control. Fusion 41, A683 (1999). 